\title{
Morale et Religion dans les manuels de français au Portugal au tournant du XIXe siècle
}

\author{
Maria José Salema
}

\section{(2) OpenEdition \\ 12 Journals}

\section{Édition électronique}

URL : https://journals.openedition.org/dhfles/2058

ISSN : 2221-4038

\section{Éditeur}

Société Internationale pour l'Histoire du Français Langue Étrangère ou Seconde

Édition imprimée

Date de publication : 1 juin 2001

Pagination : 73-85

ISSN : 0992-7654

\section{Référence électronique}

Maria José Salema, « Morale et Religion dans les manuels de français au Portugal au tournant du XIXe siècle ", Documents pour l'histoire du français langue étrangère ou seconde [En ligne], 26 | 2001, mis en ligne le 04 septembre 2014, consulté le 27 mai 2021. URL : http://journals.openedition.org/dhfles/ 2058

Ce document a été généré automatiquement le 27 mai 2021.

\section{(c) SIHFLES}




\title{
Morale et Religion dans les manuels de français au Portugal au tournant du XIXe siècle ${ }^{1}$
}

\author{
Maria José Salema
}

\section{De nouveaux manuels pour un enseignement moderne des langues vivantes}

1 Les manuels d'enseignement de la langue vivante de plus grande diffusion au XIXe siècle, le français, sont l'un des miroirs les plus intéressants des croyances véhiculées par les idéaux éducatifs alors dominants. En effet, ces outils reflètent assez fidèlement l'évolution des publics scolaires, des objectifs et des méthodes d'apprentissage conçus pour répondre aux nouveaux besoins sociaux et éducatifs qui se manifestent à cette époque. Je me suis donc proposée d'analyser dans la présente étude l'influence des valeurs morales et religieuses sur l'élaboration des manuels d'enseignement du Français Langue Étrangère utilisés dans les lycées portugais entre 1880 et 1910, dates, respectivement, du début de la remise en cause de la méthodologie traditionnelle de l'enseignement des langues vivantes, et de la fondation de la République.

2 L'une des conséquences du mouvement de rénovation pédagogique européen, qui culmine en 1882 avec la publication, en Allemagne, du pamphlet de Wilhelm Vietor: « Der Sprachunterricht muss umkehren » [L'enseignement des langues doit faire volteface], est la valorisation du 'texte suivi', par opposition à celle du mot isolé, et l'adoption, à côté des grammaires traditionnelles, d'un nouveau type de manuel scolaire, les recueils de textes ou anthologies.

3 La divulgation, dans le dernier quart du XIXe siècle, de conceptions éducatives nouvelles et la définition d'objectifs spécifiques pour l'apprentissage des langues vivantes, conciliant objectifs formatifs et culturels, d'une part, et objectifs pratiques, de l'autre, attirent l'attention des éducateurs sur le besoin de la formation encyclopédique des jeunes et sur l'indispensable étude de la civilisation des peuples dont on apprend la 
langue, et expliquent l'intérêt des enseignants au débat concernant l'adéquation des manuels à ces idéaux.

\section{Le corpus étudié}

4 Nous considérerons un ensemble de neuf anthologies destinées à la lecture et à la traduction, qui furent approuvées officiellement pour les lycées portugais entre 1880 et 1909, à savoir:

5 1. Lectures morales et morceaux choisis des classiques français /Nova selecta franceza ou Trechos extrahidos dos melhores classicos francezes em prosa e verso para o estudo da lingua franceza..., de Jacob Bensabat (Porto, Livraria Portuense de Lopes \& C.a, terceira edição, correcta e muito melhorada, 1887) ${ }^{2}$

6 2. Selecta franceza para uso dos Lyceus..., de Bernardo V. Moreira de Sá (Porto, Livraria Universal de Magalhães \& Moniz, Editores, 1882);

7 3. Selecta franceza ou Trechos extrahidos dos melhores autores francezes em prosa e verso para uso dos que aprendem a lingua franceza..., de J.-I. Roquette, nova ed. rev. e aum. por Leopold Marcou (Paris-Lisboa, Guillard Aillaud \& C.ia, s.d.);

8 4. Primeiras licções de traduccão da lingua franceza, de Domingos de Azevedo (Lisboa, Livraria de Antonio Maria Pereira-Editor, 1897);

9 5. Selecta de autores franceses- Prosa e poesia, de João Chèze, acompanhada de notas de A.R.Gonçalves Vianna (Paris-Lisboa, Guillard Aillaud \& C.ia, 1897);

10 6. Livre de lecture française à l'usage des classes de Ie, de IIe et de IIIe, de J. J. Teixeira Botelho (Porto, Librairie Char-dron de Lello \& e Irmão, éditeurs, 1907);

11 7. Lectures scientifiques et littéraires à l'usage des classes de IVe et Ve, de J.J. Teixeira Botelho (Porto, Librairie Chardron-Lello \& Irmào, éditeurs, 1909);

12 8. Livro de leitura francesa para a 1..$^{a}$, 2. ${ }^{a}$ e $3 .^{a}$ classes dos liceus, de Paul Querette (Porto, Magalhães \& Figueiredo, Limitada-Editores, 1909);

13 9. Lectures pratiques de langue française conformes aux programmes des I.II.et III classes $d u$ cours des lycées, de Júlio Monteiro (Paris-Lisboa, Aillaud \& C.ia, 1909).

\section{Les manuels de 1880 à 1907}

14 Dans leur ensemble, et jusqu'à 1907, année à partir de laquelle de nouveaux auteurs tenteront de mettre en application les principes de la méthode directe proposée par la réforme de l'enseignement secondaire de 1905, ces manuels obéissent encore aux objectifs de l'éducation humaniste classique, malgré une influence croissante du positivisme qui se manifestera dans l'introduction progressive de connaissances pratiques et de thèmes de divulgation scientifique.

\subsection{Faire connaître et admirer les grands hommes pour inciter à la pratique de la vertu}

Littérature, Histoire et Morale s'allient dans les cinq premiers manuels pour présenter aux jeunes lycéens un vaste tableau, où personnages, souvent idéalisés - les grands hommes, et événements, fréquemment héroïques, témoignent d'une vision du monde où le passé 
est proposé comme modèle pour l'action humaine. Ces textes sont rédigés, dans la plupart des cas, dans un style littéraire. Explicitement ou implicitement, qu'il s'agisse de Sentences ou de Maximes, de dialogues, de contes, de fables, d'anecdotes, de récits ou d'historiettes, la morale est le fil conducteur de la formation proposée aux jeunes. Toutefois, le cadre reste celui des objectifs généraux de l'enseignement secondaire traditionnellement poursuivis, et définis avec précision vers la fin du XIXe siècle par la réforme des lycées de 1894. Si les objectifs du nouveau programme de français sont l'étude de la littérature classique et de la langue et de la civilisation françaises, les normes pédagogiques établies par le Règlement Général de l'Enseignement Secondaire de 1895 soulignent l'importance de l'éducation morale des lycéens: «Toutes les activités scolaires, notamment le contenu éthique des cours des différentes matières ", constituent des moyens efficaces pour atteindre le but le plus général de l'enseignement des lycées qui est « le développement moral des élèves ».

De même, les auteurs de nos anthologies soulignent dans leur préface que ce qui les a guidés dans l'élaboration de ces outils didactiques, c'est l'apprentissage et le perfectionnement linguistique des élèves, le développement de leur " goût littéraire ", leur motivation à la lecture, lecture qui devra instruire, plaire et amuser, et leur formation morale. Parmi les Morceaux choisis des classiques français, qui constituent la deuxième partie du manuel de Bensabat, un texte de M. Saint-Marc Girardin, L'instinct et le sentiment en littérature, préconise pour la littérature un rôle moral semblable à celui de la famille, source d'éducation morale, qui « aboutit à la plus pure des morales, la piété filiale »: (Bensabat $1887: 194)$ :

Je demande à la littérature d'imiter cet ordre divin et de donner à l'expression des affections naturelles à l'homme la beauté morale qu'elles doivent avoir.

L'histoire a une mission semblable, comme le souligne Rollin dans le texte L'Histoire, (cité par Chèze $1897: 143$ ) :

L'histoire, quand elle est bien enseignée, devient une école de morale pour tous les hommes. Elle décrie les vices, elle démasque les fausses vertus. [...] et démontre par mille exemples, plus persuasifs que tous les raisonnements, qu'il n'y a de grand et louable que l'honneur et la probité. [Enfin] elle fait conclure que la vertu est le véritable bien de l'homme, et qu'elle seule le rend véritablement grand et estimable.

Les beaux textes d'histoire qui remplissent nos manuels : portraits, narrations, récits historiques, caractères, traits historiques, immense galerie où se côtoient rois, empereurs et princes, personnages illustres de tous les degrés de l'échelle sociale, de la noblesse, de l'Église (papes, évêques, curés, saint et saintes), des arts (peintres et musiciens), des lettres, poètes et philosophes notamment, de l'armée (chefs militaires, mais aussi braves soldats), nous y sont généralement présentés comme " exemples sublimes de vertus ». La place des Anciens, les Grecs et les Romains surtout, y est considérable. Quelques intitulés et quelques épithètes, illustrant des qualités, des sentiments, des facultés, des vertus, parlent d'eux-mêmes. Louis XIV et Marie Antoinette sont " deux augustes personnages ", exemples de " sensibilité »; Alexandre, 1'« homme qui a ressemblé à un dieu parmi les hommes "; Louis de Bourbon, comte de Montpensier, "le héros de la tendresse familiale »; Cicéron et Démosthènes, "les plus grands orateurs de l'antiquité ». Dans Éponine et Sabinus, la première est le "modèle d'épouse dévouée et vertueuse » et 1'« héroïne de l'amour conjugal »; Descartes est présenté comme le " génie puissant et hardi »; quant à Beethoven, il est dit être dans La mort de Beethoven, " le vrai prophète de Dieu ». 
Développer la capacité de compréhension des œuvres les plus importantes de la littérature classique du XVIIe au XIXe siècle chez les jeunes élèves de français, mais aussi faire connaître et admirer les grands hommes, émouvoir, pour inciter à la pratique de la vertu, fondement et finalité première de l'éducation, voilà le but de la lecture de ces textes. Pour Roquette (s.d.), par exemple, le mot Éducation, titre de l'un de ses textes signifie formation morale de l'homme, tandis qu'Azevedo (1897 : V-VI) nous informe qu'il a visé, non seulement à « rendre facile la compréhension des thèmes choisis ", mais aussi à « insinuer des idées morales, religieuses et instructives, capables de laisser une trace salutaire dans l'esprit des enfants ». Enfin, en soulignant l'importance de la lecture fréquente, G. Viana, co-auteur du manuel de Chèze déjà cité (1897 : VIII), justifie la « sélection scrupuleuse " qui l'a guidé pour ce qui est la « moralité du conte ", que « la perspicacité naturelle des élèves saura déduire ", au cas où elle ne serait pas « clairement indiquée ».

\subsection{La religion la seule base solide de la vertu}

Cette morale, centrée sur la vertu, au cœur de toute l'activité humaine, est une morale exigeante et austère pour les jeunes, étroitement associée à la religion: « c'est de la morale religieuse qu'elle tire ses plus puissants auxiliaires " (Roquette s.d. : 83), car (Azevedo1897:4) :

la seule base solide de la vertu est la religion, et le premier principe de la religion est la croyance en Dieu.

21 Cette morale s'inscrit dans le cadre d'une histoire proche de la conception de Bossuet, dont Dieu est le moteur et qui est destinée à montrer « l'action de la Providence dans le développement de l'humanité » (Cf. Giolitto 1984 : 134-135). En effet, c'est Dieu qui guide l'homme dans la marche de la civilisation, qui est une (Azevedo, ibid. : 82)

marche vers le progrès, vers la vertu et tous les développements des meilleures facultés de l'homme.

Le premier devoir de l'homme est, donc, d'adorer Dieu, d'adorer « l'auteur de la nature ", dont on contemple " les admirables spectacles, les merveilleuses harmonies : la nature est le trône extérieur de la magnificence divine " (ibid. : 1). Tout dans l'univers, nature et culture, révèle le pouvoir créateur de Dieu, sa sagesse et sa prévoyance. La science, elle-même, " en découvrant les secrets de la nature, ne fait que nous révéler chaque jour davantage la grandeur de Dieu qui l'a créée » (ibid. : 73).

Il s'agit là d'une religiosité romantique, que de nombreux textes de Lamartine, Chateaubriand Victor Hugo, Musset, Bernardin de Saint Pierre et autres prosateurs et poètes attestent dans leurs descriptions ou évocations: le lever et le coucher du soleil, la mer, orages, tempêtes, ouragans, forêts, montagnes, lacs, fleuves, déserts, villes anciennes et modernes, catacombes, ruines, promenades et voyages. La beauté de la liturgie catholique touche aussi la sensibilité (Azevedo, ibid. : 92) :

La religion chrétienne, dans ses cérémonies parle à la fois à l'âme et aux sens.

\subsection{L'utilité de la religion}

Un certain utilitarisme se mêle souvent à cette religiosité. La religion est plutôt l'instrument d'une sagesse pratique, d'une morale de prudence sociale, utilitaire. On peut ainsi lire dans l'anthologie d'Azevedo (ibid. : 91) : 
Le sentiment religieux est le plus utile de tous, peut-on lire, car il crée tous les bons sentiments, les stimule, les dirige, en même temps qu'il comprime tous les mauvais. - Sans la religion, la vieillesse n'a pas d'avenir, elle voit arriver avec effroi le moment de la mort... avec la religion, au contraire, elle attend la fin de la vie avec une douce résignation, je dirai même avec bonheur. essentiellement une morale du devoir, de l'effort, du travail, du sacrifice, de l'héroïsme, de l'obéissance. Parmi les 423 phrases destinées à la traduction, qui constituent la première partie du manuel d'Azevedo, plusieurs concernent le devoir. Nous n'en citerons que la quatre-vingtième (ibid. : 13) :

Le devoir est la loi divine, morale et humaine en action, c'est le bonheur unique et suprême, c'est la règle sacrée de tous.

Devoir envers Dieu, auquel il faut se soumettre mais aussi envers la famille, qui est la « première école de toutes les vertus » où l'enfant est " armé pour les luttes de la vie » (Bensabat 1887 : 267); enfin, devoir envers les parents (Lamennais cité par Moreira de Sá $1882: 22)$ :

Enfants, apprenez quels sont vos devoirs envers vos parents; car vous ne serez heureux et bénis qu'en y restant fidèles.

le principal élément de l'union, du bonheur et de la force des nations... et la base de tous les gouvernements.

veille sur les crimes privés, [les lois veillant sur] les crimes publics.

n'est pas seulement la meilleure des religions [mais] la meilleure des philosophies, mettant toutes les vertus, toutes les générosités, tous les mérites au concours. que les thèmes religieux occupent dans les manuels et des intitulés comme: La religion, Le christianisme, La Providence, Magnificence et bonté de Dieu dans la nature, Adoration, L'existence de Dieu, La Prière, Le Pater, Aspiration au ciel, Triomphe de la charité et de la modestie. Réflexions sur l'écriture sainte, L'aumône, etc.

\subsection{Une morale du devoir}

La piété familiale » est, en effet, la plus pure des morales, « le premier article de notre code moral et religieux » (Roquette s.d. : 29).

De nombreux textes présentent aux jeunes apprenants de français des exemples tirés de l'histoire, tout un ensemble de vertus, caractères, qualités, sentiments, dont on souligne la bonté/charité/compassion/bienfaisance, générosité, qui peuvent aller jusqu'au dévouement. Roquette disait encore (ibid. : 78-79) :

[le dévouement] consiste à braver la mort pour le salut de ses semblables, à prendre une détermination qui peut coûter la vie, mais qui amènera des résultats utiles à sa famille, à ses amis, à sa patrie,

Quant à l'amour de la patrie (ibid. : 40),

[il est] le moyen le plus efficace qu'il faille employer pour apprendre aux citoyens à être bons et vertueux, 
colets de l'obéissance: il y a, en effet, d'une part, " l'obéissance d'amour, l'amour filial ou fraternel dans toute sa beauté »; de l'autre, « l'obéissance de l'honneur ", c'est-à-dire, "l'obéissance patriotique " (Chèze 1897 : 140-141). Cette dernière appartient aux « devoirs de société » auxquels l'écolier doit aussi se préparer, puisque, "fait pour la société ", l'homme " est né pour créer et braver le canon " (Bensabat 1887 : 173-174). À l'instar de l'enfant de 13 ans, tué par les royalistes en 1793, que Victor Hugo célèbre dans sa poésie À la barricade, notre jeune écolier peut, lui aussi, devenir héros. Et si Dieu imposa « la loi du travail », puisque " l'homme n'a rien sans peine, si le travail est nécessaire, si le travail ennoblit l'homme, les fruits de l'étude " sont évidents : son utilité ne se borne pas à la science, elle donne aussi la capacité pour les affaires et pour les emplois. De plus, l'étude fait acquérir l'amour du travail, elle en adoucit la peine » (Moreira de Sá $1882: 79$ ).

Le loisir n'a pas de place dans la vie des écoliers. On leur conseille, donc, de travailler sans repos, de ne pas oublier que (Bensabat $1887: 1$ ):

la jeunesse est le temps de semer : Ne perdez pas de temps à des choses frivoles, et appliquez-vous à acquérir les connaissances nécessaires pour remplir la mission que la Providence vous a désignée... Profitez des leçons que vous recevrez pour devenir vertueux et instruits. Aimez le travail.

Le temps libre sera consacré " à la lecture de bons livres ", qui élèvent " l'esprit » et inspirent « des sentiments nobles et courageux » (Azevedo 1897 : 66). En effet, la lecture est « le plus utile des amusements» (Bensabat $1887: 1$ ).

37 A côté des Sentences et des Fables, formant généralement une partie indépendante de ces anthologies, on trouve aussi, dans ces dernières, des dialogues, entre l'écolier et l'adulte, le père, l'instituteur, mais surtout la mère. Ces conversations véhiculent des notions, des connaissances ou des normes de conduite sociale qui sont tirées soit de récits ou de contes, soit de leçons de choses de la vie quotidienne, soit de la réflexion sur l'histoire passée soit de l'observation de l'environnement de l'écolier.

Cet enseignement prend souvent la forme de conseils, d'exhortation à une pratique positive des vertus, ou de prévention contre les conséquences qui pourraient être fâcheuses de leur mépris ou de l'attrait des vices:

«Mes enfants, il faut qu'on travaille » (Moreira de Sá 1882 : 294);

«Oh! Soyons bons premièrement, et puis nous serons heureux» (Bensabat 1887 : 132).

«L'ambition est la plus vorace des passions » (Azevedo 1897 : 69);

«l'orgueil est une folie de l'esprit...il peut être une cause de démence, même physique » (ibid. : 73);

3 « le luxe est la plaie des familles et des nations » (ibid. : 75).

\subsection{Des contenus en évolution}

Il n'y a pas d'évolution linéaire dans les manuels analysés. Malgré les caractéristiques communes qu'on vient de déceler dans tous ceux qui furent utilisés jusqu'à 1907, certains présentent déjà des nouveautés dans leurs contenus. Tel est le cas de l'œuvre de Moreira de Sá qui met en évidence la réduction du nombre de textes historiques, surtout de ceux qui portaient sur l'Antiquité et une place plus large réservée aux pages des écrivains contemporains et au français non littéraire. En somme, il s'agit d'un monde plus moderne et plus ouvert offert aux jeunes lecteurs, qui introduit les 
découvertes scientifiques et techniques récentes, traduit l'éblouissement devant les progrès de l'industrie, constituant l'embryon des futurs manuels, véritables encyclopédies fourre-tout, qui intégreront les domaines les plus divers: géologie, astronomie, musique, économie, cosmographie, sciences naturelles, anthropologie, arts, physique, chimie, physiologie, etc. Quelques titres illustreront cette nouvelle optique :

La fabrication des épingles [exemple des] prodiges créés par la division du travail,

Les manufactures des États-Unis,

Une mine de fer en Suède,

Les races et les religions de l'Europe,

Les peuples éclairés,

Le premier bateau à vapeur...

\section{Les manuels de 1907 à 1910}

\subsection{Un enseignement des langues plus utilitaire}

En 1907 et en 1909 le gouvernement approuve quatre nouveaux manuels pour l'enseignement du français, en accord avec la méthode directe récemment proposée (on sait que les fondements de cette dernière sont la priorité de l'oral, l'apprentissage inductif de la grammaire et l'adoption du texte suivi), et avec les nouvelles normes sur l'élaboration des livres destinés à la lecture, qui est désormais l'activité centrale de la classe. Cette méthodologie aura pour but d'initier l'élève à la langue et la civilisation françaises, d'abord par l'étude de textes qui, d'une part, sont faciles, d'autre part, présentent des " connaissances pratiques sur les sciences, l'art, les industries et le commerce ", et des " récits de voyage ", mais aussi des extraits d'ouvrages historiques et des poésies des "différents genres et époques de la littérature française ». Sous l'influence de 1'aspiration de l'opinion publique à un enseignement des langues plus utilitaire, le législateur de 1905 insiste maintenant moins sur la formation humaniste de cet enseignement que sur sa finalité pratique de langues de communication. Dans les préfaces des nouveaux livres de lecture, leurs auteurs ne soulignent que leur intention d'être fidèles aux objectifs pédagogiques des nouvelles directives officielles.

\subsection{D'une morale d'inspiration religieuse à une morale humanitaire et civique}

Si la morale traverse encore la plupart de ces textes, ce n'est plus une morale formelle et d'inspiration religieuse. À la transmission des valeurs morales traditionnelles qui exaltent la famille, le devoir, l'obéissance, le travail, l'étude, le courage et l'abnégation, l'amour de la patrie, s'ajoute l'apprentissage des règles de l'hygiène, de la civilité, de la politesse, de l'éducation civique. L'élève devra, non seulement apprendre à parler et à écrire le français, à connaître la France dans sa géographie et dans son histoire, dans ses institutions et dans ses mœurs, mais acquérir les connaissances usuelles dont il aura besoin lorsqu'il exercera un métier et apprendre les préceptes de la morale humanitaire et civique (Cf. Giolitto $1984: 64$ ). Voici quelques nouveaux exemples des nouveaux thèmes et titres :

La fête de l'aïeule,

Entretien de la maison et des vêtements, 


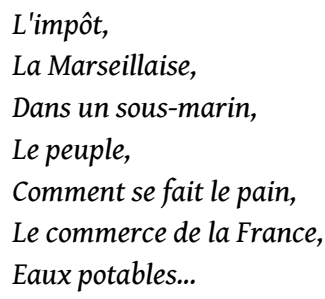

47 Si le style des textes qui présentent les connaissances scientifiques est presque toujours descriptif, les nouvelles notions sont généralement transmises dans des fables, des leçons de choses, des historiettes, des anecdotes, des contes parfois amusants, tous des textes courts et rédigés dans un style assez simple.

L'apprentissage s'articule maintenant autour des centres d'intérêt : la maison et la vie familiale, le corps humain, l'école et l'étude, les vêtements, la France et les habitudes des Français, les personnages les plus emblématiques de l'histoire de France, la vie en France, surtout à la campagne, aliments, fruits et animaux...

L'univers religieux disparaît presque totalement de ces livres, à l'exception de quelques poésies où Dieu, la Vierge et les saints sont évoqués.

\section{Conclusion}

L'analyse des manuels de français que nous venons de présenter permet de constater l'importance et, en même temps, la permanence de la composante morale dans leurs contenus. Celle-ci est l'un des axes majeurs de l'éducation des élèves portugais. Notre analyse permet également de vérifier qu'il se produit une évolution: on passe, en effet, d'une morale d'inspiration religieuse, explicitement et implicitement omniprésente, à une morale laïque et pratique, dont l'une des dimensions est l'éducation civique.

51 Cette première recherche devrait pourtant être approfondie. Il faudrait compléter l'étude des manuels d'enseignement de la langue maternelle, avec d'autres données comme la connaissance de l'idéologie des auteurs ou la question plus générale de l'éducation morale et religieuse des jeunes, dans le contexte politique et social de la société portugaise du tournant du siècle.

\section{BIBLIOGRAPHIE}

CARAVOLAS, Jean-Antoine. 1995. Le point sur l'histoire de l'enseignement des langues (3000-1950). Centre Educatif et Culturel Inc. Anjou.

CLEMENTE, Manuel. 1994. « Igreja e Sociedade Portuguesa do Liberalismo à República », Didaskalia, vol. XXIV fasc. 1: 119-129.

GIOLITTO, Pierre. 1984. Histoire de l'enseignement primaire au XIXe siècle, vol. II: Les méthodes d'enseignement. Paris: Nathan. 
MORAZË, Charles (dir.). 1968. Histoire du développement culturel et scientifique de l'humanité, vol. V, Le XIXe siècle, T. I, Paris: Robert Laffont.

SALEMA, Maria José. 1993. A didáctica das linguas vivas e o ensino do francés nos liceus portugueses na viragem do século : o período de 1894 a 1910. Braga: Universidade do Minho (Tese dactilografada).

SERRÃo, Joel (Org.). 1963-1971. Dicionário de Historia de Portugal. Vol.III. Lisboa: Iniciativas Editoriais.

\section{NOTES}

1. La thématique de ce texte, remanié, fut l'objet d'une communication à la National University of Ireland, lors de la XIXth International Standing Conference for the History of Education, qui s'est tenue à Maynooth, en septembre 1997, sous le titre «Faiths and Education : Historical and Comparative Perspectives ».

2. Une première édition de cet ouvrage (1881) fut aussi approuvée en 1882.

\section{AUTEUR}

\section{MARIA JOSÉ SALEMA}

Universidade do Minho 